\title{
A Novel Pattern Classification using Granular Reflex Fuzzy Min-Max Neural Network
}

\author{
Ramesh Kakollu \\ M.Tech Student \\ Gudlavalleru Engineering College,Gudlavalleru \\ Krishna Dist, A.P., India, Pincode-521356
}

\author{
E. Vargil Vijay \\ Assistant Professor \\ Gudlavalleru Engineering College,Gudlavalleru \\ Krishna Dist, A.P., India, Pincode-521356
}

\begin{abstract}
Pattern classification is a system for classifying patterns into dissimilar potential categories. The classifier that is used for classification is granular neural network. A granular neural network called granular reflex fuzzy min-max neural network (GrRFMN). GrRFMN uses hyperbox fuzzy set to signify grainy information. Using known data the neural network will be trained, and using this trained neural network data can be classified. Its structural design consists of a spontaneous effect system motivated from human brain to handle group overlies.

The GFMN cannot hold data granules of dissimilar sizes professionally. It can be practically done that a convinced quantity of such preprocessing can assist to recover the presentation of a classifier. The GrRFMN is skilled of managing grainy information capably by the training algorithm. The experimental outcomes on valid datasets confirm a good presentation of GRFMN. Experimental results on valid data sets confirm that the GrRFMN can categorize granules of dissimilar granularity further acceptably.
\end{abstract}

\section{Keywords}

Compensatory neurons, grainy information classification, granular neural network (GNN), reflex mechanism.

\section{INTRODUCTION}

Pattern identification is a method which resembles the same nature as human opinion, knowledge, and analysis. Normally, calculation is nothing but handling of numbers or symbols. Even though, major evolution in the field of video processing, human recognition can always disagree with system based pattern identification. It is a pattern identification crisis to study and organize grainy information. The supervised and unsupervised learning means the essential arrangement of the data is to be capable by GRFMN. In pattern recognition systems, partially supervision for the practical execution is of more significance. Computers cannot tackle the complex arrangement at rest, while humans can appear better tasks to solving. Even if, the non-numeric cluster of data (information granules) during adding just before individual numeric values are based on aptitude of human identification [1]. The invisible things of the space form clusters and drawn simultaneously by various parallel of identical objects called granules [2]. Another way, one of many narrow frequency sub bands, the arrangement of which composes an entire signal and each sub band carries its own specific data [3]. The hyper boxes and intervals are formed by information granule represents simple and straightforward way. The granular selforganizing map (grsom) detect clusters in the information and situate fuzzy interval numbers (FIN) on each cluster so it covers entire data, overlie will occur. GrRFMN undertake the overlie problem by using reflex mechanism in neural network algorithm. This project also addresses a problem to treat the working out information and study from the previous data. It is practical that such a preprocessing of information be capable of progress presentation of a classifier. The future novel education approach to contract with moderately labeled information and insertion of compensatory neurons has enhanced the presentation of GRFMN extensively. Though calculating a novel group otherwise a removed group can be capable while learning the data in a particular way to navigate and unnecessary of retraining in GRFMN. The identifying limits can take several authentic humankind circumstances in which individual desires by the kind of granules. Applications of information granulation in different sectors are laser diffraction to optimize and control, sensor data mining, computer intelligence, medical risk pattern identification in the encephalopathy.

\section{LITERATURE SURVEY 2.1 FMNN (Fuzzy Min-Max Neural Network)}

The underlying structure of information is learned by FMNN to providing hyper boxes for each class. Expansion coefficient $(\Phi)$ is the factor which can organize the maximum hyper box size during learning period. The reduction method can eliminate the overlie formed amongst hyper boxes belonging to several classes. The disadvantage of FMNN algorithm is the expansion coefficient is mostly dependent in initialization process.

\subsection{FMCN (Fuzzy Min-Max Neural Network with Compensatory Neurons)}

The FMCN is a significant classifier supports on hyper box fuzzy set and compensatory neurons. The hyper box overlies and containments are handled by the FMCN. By adding of CNs the FMCN can improve their performance. The complexity of FMCN learning algorithm is less when contrast to FMNN and GFMN. The reflex mechanism of human brain inspires the CNs and information will pass through in particular way and learning will be simple manner.

\section{A NOVEL GRRFMN ARCHITECTURE}

The novel design of GrRFMN is publicized in Fig. 1 and is depends on the FMCN [2]. The problem of the network is handled during training phase. All the input characteristics are scaled in $[1,0]$. The hyperbox lower and greater indicate vectors $(2 n)$ are associated to the input nodes $b_{h 1}-b_{h 2 n}$. The quantity of characteristics used for difficulty is same as twice the number of input nodes.

The central layer neurons and output layer nodes are divided into two parts: neuron part classifier and reflex part. The reflex part is again separated into overlie compensation neuron $(\mathrm{OCN})$ part and control compensation neuron $(\mathrm{CCN})$ part. 
The lower (A) or greater (B) points are the position shows the interaction between input and hyperbox node in the center layer. In the center layer, the functions of neurons are described. The reflex part represents the group overlies and controls happening in the neuron part classifier. Throughout the training process the neurons in the center layer are active.

Contrast to the design in [2], the dual connection from an input to classifying and compensation nodes, which can signify the hyperbox lower-greater points, is changed by a particular connection. The input nodes are connected to the hyperbox nodes by a single connection. The point data and granular data are handled easily by the OCN, $\mathrm{CCN}$, and neuron section classifier. The overlap region belongs only when hyperbox granule, $\mathrm{OCN}, \mathrm{CCN}$ are stimulated.

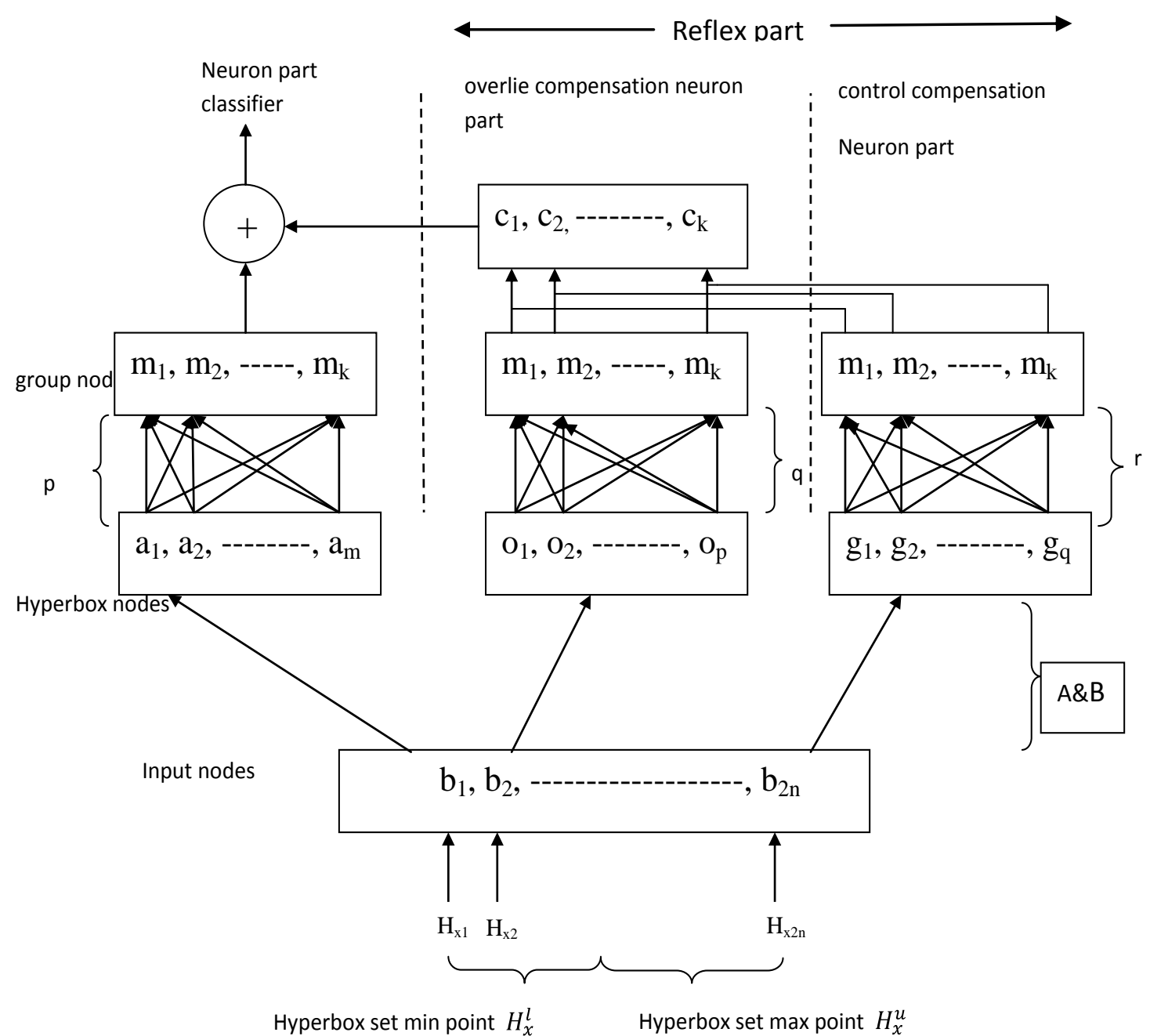

Fig.1.g $\mathrm{g}_{1}-\mathrm{g}_{\mathrm{q}}$ : control compensation hyperbox nodes; and $\mathrm{c}_{1}-\mathrm{c}_{\mathrm{k}}$ : general compensation nodes architecture where $H_{\mathrm{x}}=\left[\mathrm{H}_{\mathrm{x}}{ }^{\mathrm{u}}, \mathrm{Hxl}\right]$ : input granule; $b_{1}-b_{n}$ : input nodes; $a_{1}-a_{m}$ : arrangement hyperbox nodes; $d_{1}-d_{k}$ : group nodes; $o_{1}-o_{p}$ : overlie

\section{GRRFMN MEMBERSHIP CRITERIA}

The ratio of overlie area in the HFS and IG along dimension to the region of IG beside every measurement for the connection computation as publicized in fig. 2 are specified by means of

$\mathrm{u}=\frac{1}{\mathrm{n}+1} \sum_{\mathrm{i}=1}^{\mathrm{n}+1}\left[\begin{array}{c}\text { along ith parameter the overlie region among } \\ \text { input granule and HFS } \\ \begin{array}{c}\text { along ith parameter the whole region below } \\ \text { input granule }\end{array}\end{array}\right]$

Where $\mathrm{n}$ is the no. of measurements.

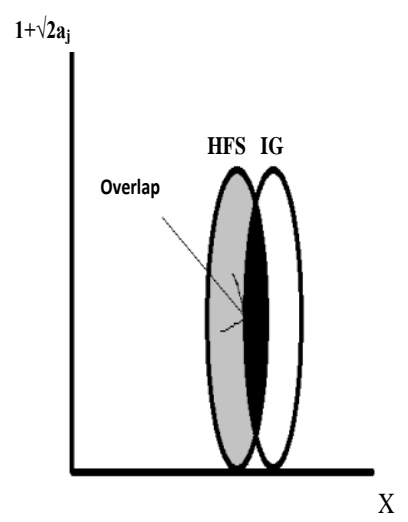

Fig.2. membership computation 


\section{ALGORITHM}

There are two paces in this algorithms, hyperbox formation/extension and compensatory neuron formation. Suppose with the purpose of the controlled couple $\left\{\mathrm{H}_{\mathrm{x}}, \mathrm{d}_{\mathrm{i}}\right\}$ signifies a learning example $\mathrm{H}_{\mathrm{x}} \in \mathrm{d}_{\mathrm{i}}$ and also $\left\{\mathrm{p}_{\mathrm{j}}, \mathrm{d}_{\mathrm{k}}\right\}$ signifies a hyperbox $p_{j} \in d_{k}$, and maximum hyperbox range is restricted by the extension coefficient $\left({ }^{\beta}\right)$.

Earlier the system by a hyperbox $\mathrm{a}_{1}$ among $\mathrm{A}_{1}=\mathrm{H}_{\mathrm{x}}{ }^{1}, \mathrm{~B}_{1}=\mathrm{H}_{\mathrm{x}}{ }^{\mathrm{u}}$ and group tag $d_{i}$ for a controlled couple of information $\left\{H_{x}\right.$, $\left.\mathrm{d}_{\mathrm{i}}\right\}$; replicate the subsequent paces $\mathrm{i}$ and ii on behalf of all learning particles otherwise tests.

STEP i: Formation/Extension

$$
\begin{aligned}
& A_{d i}=\operatorname{greater}\left(A^{\prime}{ }_{k i}+\sqrt{ } 0.2, A^{\prime}{ }_{j i}+\sqrt{ } 0.2\right) \\
& A_{d i}=\operatorname{lower}\left(B^{\prime}{ }_{k i}+\sqrt{ } 0.2, B_{j i}^{\prime}+\sqrt{ } 0.2\right)
\end{aligned}
$$

Else hyper boxes are similar control

Go to step ii(c)

b) Overlie compensation:

The greater and lower points of hyperbox are used to generate an $\mathrm{OCN}$

$$
A_{o c i}=\text { greater }\left(A^{\prime}{ }_{k i}+\sqrt{0} 0.2, A_{j i}^{\prime}+\right.
$$

The novel learning information is holded by obtain a explore intended by offering hyperbox $a_{j}$ of group $d j=d_{i}$ present the maximum connection and a novel learning information particle $\left\{\mathrm{H}_{\mathrm{x}}, \mathrm{d}_{\mathrm{i}}\right\}$

a) The $\mathrm{OCN}$ otherwise $\mathrm{CCN}$ was unrelated by this learning information

$r\left(\operatorname{sum}\left(\operatorname{greater}\left(B_{\mathrm{ji}}^{\prime}+\sqrt{0} 0.2, H_{x i}^{u}\right)-\left(\operatorname{lower}\left(A_{j i}^{\prime}+\sqrt{ } 0.2, H_{x i}^{l}\right)\right)\right) \leq \beta\right.$

Adjust the greater $\&$ lower points of hyperbox $\mathrm{p}_{\mathrm{j}}$ as, for $\mathrm{i}=1$ to

$$
\begin{aligned}
& A_{j i}^{\text {new }}=\operatorname{lower}\left(A_{j i}^{\prime \text { old }}+\sqrt{0} 0.2, H_{x i}^{l}\right) \\
& B_{j i}^{\text {new }}=\operatorname{greater}\left(B_{j i}^{\prime \text { old }}+\sqrt{ } 0.2, H_{x i}^{u}\right)
\end{aligned}
$$

Go to step ii

If a proper $a_{j}$ is not initiate, create a new hyperbox with $A_{j}=$ $\mathrm{H}_{\mathrm{x}}{ }^{1}, \mathrm{~B}_{\mathrm{j}}=\mathrm{H}_{\mathrm{x}}{ }^{\mathrm{u}}$ and group $\mathrm{d}_{\mathrm{i}}$.

Go to step i.

\section{STEP ii: Compensatory Neuron Formation}

Imagine in earlier step the hyperbox $p_{j}$ extension with $p_{k}$ and $\mathrm{d}_{\mathrm{j}} \neq \mathrm{d}_{\mathrm{k}}$ is contrasted

\section{1) Test for isolation:}

If $\left(B^{\prime}{ }_{k i}+\sqrt{0} 0.2<A_{j i}^{\prime}+\sqrt{0.2}\right)$ or $\left(B_{j i}^{\prime}+\sqrt{0} 0.2<A_{j i}^{\prime}+\right.$ $\sqrt{0.2})$ is accurate for any $i$,

Then $\left(p_{k}, p_{j}\right)$ are inaccessible and Go to step i. Else step ii (b).

1) Test for neuron creation:

a) Control compensation:

If $\left(\mathrm{A}_{k i}^{\prime}+\sqrt{0.2}>\mathrm{A}_{j i}^{\prime}+\sqrt{0.2}>\mathrm{B}_{j i}^{\prime}+\sqrt{0.2}>\right.$ $\left.\mathrm{B}^{\prime}{ }_{k i}+\sqrt{0} 0.2\right)$ or $\left(\mathrm{A}_{j i}^{\prime}+\sqrt{0} 0.2>\mathrm{A}^{\prime}{ }_{k i}+\sqrt{ } 0.2>\mathrm{B}_{k i}^{\prime}+\right.$ $\left.\sqrt{0.2}>\mathrm{B}^{\prime}{ }_{j i}+\sqrt{ } 0.2\right)$ is accurate for any $\mathrm{j}^{\prime}(\mathrm{j} \varepsilon 1---\mathrm{n})$

The hyperbox greater and lower points are specified after that a CCN can generate with

$$
B_{o c i}=\operatorname{lower}\left(B^{\prime}{ }_{k i}+\sqrt{ } 0.2, B_{j i}^{\prime}+\sqrt{ } 0.2\right)
$$

Go to step i.

\section{EXPERIMENTAL RESULTS}

Take an $\mathrm{X}$-axis and $\mathrm{y}$-axis and values are placed on the axis. Draw the points using those values and form an overlie groups as depicted in Fig.3, which shows the ultimate membership status calculation and functioning of compensatory neurons $(\mathrm{OCN}$ and $\mathrm{CCN})$. Single OCN and dual CCNs are occurred during the over lied along with various groups.

In Table I there are four groups F1, F2, F3 and F4 have a membership of test granules G1, G2 and G3 and their compensations are specified. The group overlie regions in the compensatory neurons is reduced by the test information (e.g., G2 and G3) contribute to membership whenever the four groups of ultimate memberships are revealed in Table II.

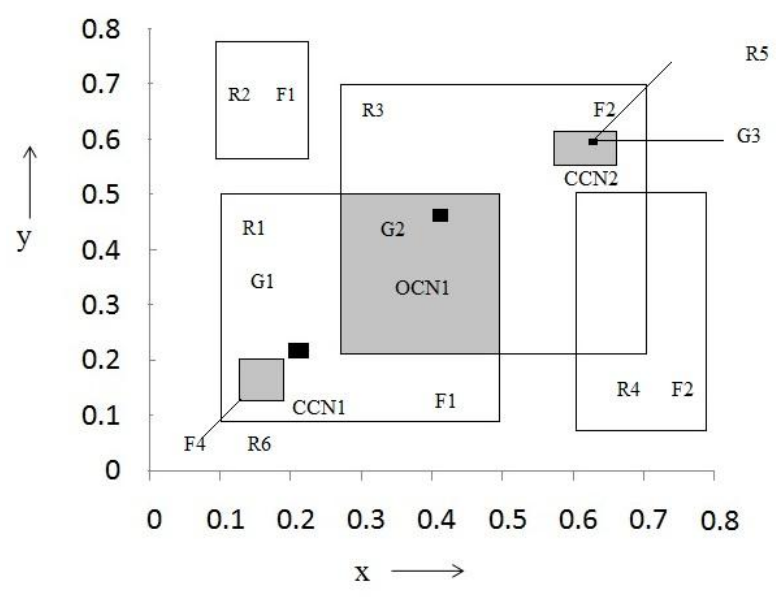

\begin{tabular}{|c|c|c|c|c|c|c|c|c|}
\hline \multirow{4}{*}{$\begin{array}{c}\text { Test Point } \\
\mathrm{P}[\mathrm{v}, \mathrm{w}]\end{array}$} & \multicolumn{6}{|c|}{ Classification section output } & \multicolumn{2}{|c|}{ Compensation section output } \\
\hline & \multicolumn{6}{|c|}{ Class } & \multicolumn{2}{|c|}{ Class } \\
\hline & \multicolumn{2}{|c|}{$\mathrm{F} 1$} & \multicolumn{2}{|c|}{$\mathrm{F} 2$} & F3 & $\mathrm{F} 4$ & $\mathrm{~F} 1$ & $\mathrm{~F} 2$ \\
\hline & R 1 & R3 & $\mathrm{R} 4$ & $\mathrm{R} 2$ & R5 & R6 & OCN1 CCN1 & OCN1 $\mathrm{CCN} 2$ \\
\hline
\end{tabular}

Fig.3.Functioning of $\mathrm{OCN}$ and $\mathrm{CCN}$, where the test granules are G1, G2, G3; and the hyperboxes are R1-R6; and groups are F1-F4; and a single OCN1 and dual CCNs (CCN1, CCN2)

Table 1 Membership Computation 


\begin{tabular}{|c|c|c|c|c|c|c|c|c|c|c|}
\hline $\mathrm{G} 1[(0.22,0.22),(0.24,0.24)]$ & 1.0 & 0.5 & 0.759 & 0.5 & 0.0 & 0.81 & 0 & 0 & 0 & 0 \\
\hline $\mathrm{G} 2[(0.35,0.37),(0.38,0.39)]$ & 1.0 & 0.35 & 1.0 & 0.25 & 0.46 & 0.0 & -0.1705 & 0 & -0.2631 & 0 \\
\hline $\mathrm{G} 3[(0.52,0.42),(0.54,0.44)]$ & 0.545 & 0.43 & 1.0 & 0.54 & 1.0 & 0.0 & 0 & 0 & 0 & -1 \\
\hline
\end{tabular}

Table 2 Final Membership after Adding Compensation

\begin{tabular}{|c|c|c|c|c|}
\hline \multirow{2}{*}{ Test point } & \multicolumn{4}{|c|}{ Class } \\
\cline { 2 - 5 } & F1 & F2 & F3 & F4 \\
\hline G1[(0.22,0.22),(0.24,0.24)] & 1 & 0.75 & 0 & 0.79 \\
\hline G2[(0.35,0.37),(0.38,0.39)] & 0.8295 & 0.745 & 0.46 & 0 \\
\hline G3[(0.52,0.42),(0.54,0.44)] & 0.545 & 0 & 1 & 0 \\
\hline
\end{tabular}

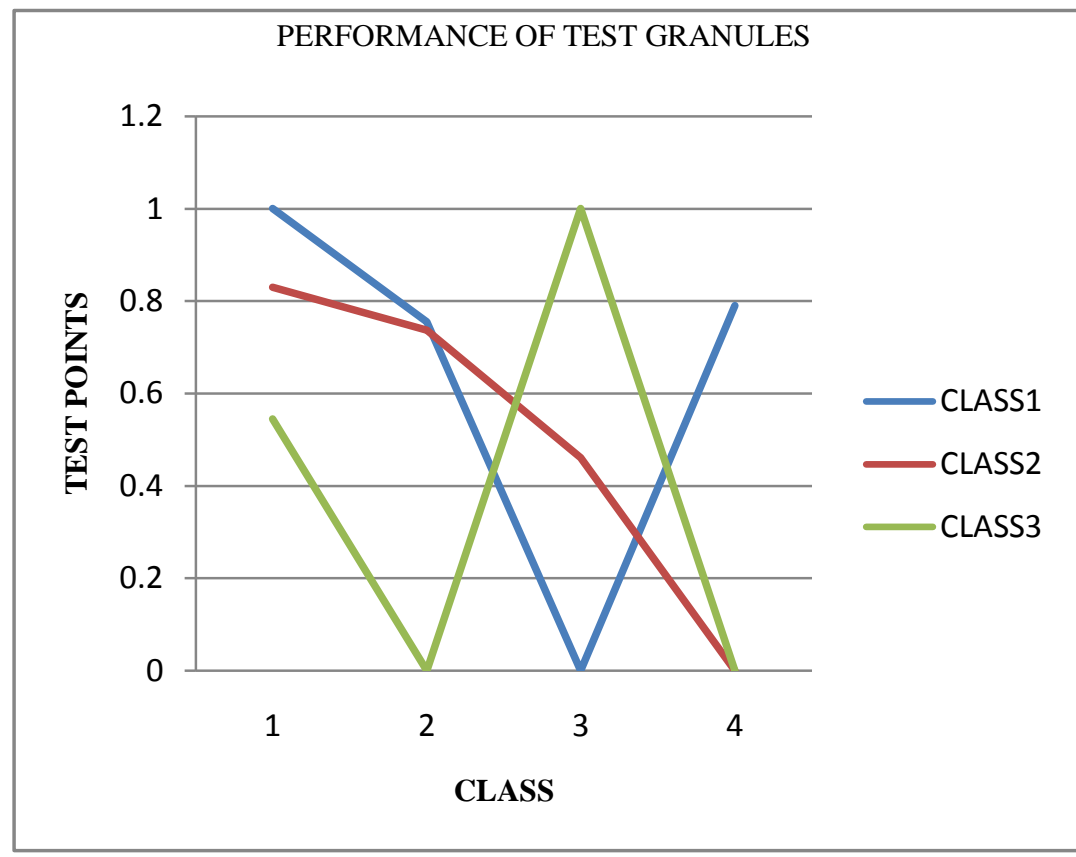

Fig.4. Performance of iris data set

In the figure.4, the performance of iris data set shows the classes in the test granules. The test points in the membership computation, the size varies from 0 to 1.2 and the class 0 to 1 . The performance of test granules can shows the class expansion. The classes 1 to 3 are to be mentioned in different colors. However, it is also observed from the results that if data is less granulated granulation and preprocessing many not be so effective.

\section{CONCLUSION}

A pattern identification crisis to be trained and also organize grainy information was considered. A qualified GrRFMN can organize granules of various sizes properly and find out crisp granule membership by the inauguration function capable of usage granular data proficiently. The overlies in granular data was hold by using GrRFMN reflex mechanism. The reflex mechanism in GrRFMN was found useful to handle overlies in granular data. It was practically suitable to inclusion of reflex mechanism, GrRFMN eliminate the basic structure in information more suitably and gives enhanced classification exactness. The grainy information is capable to activate and handled by the GrRFMN and the granular information can be trained and shows better appearance. The possibility of condensation of training data and learning from it was also explored. It was observed that a certain amount of such preprocessing can help to improve the performance of a classifier. The overlie region can be very less and it shows the patterns frequently. From the experimental outcomes for GrRFMN on valid data sets can show improvement in results by the use of granular information. This work can be applied further for color and video images for solving real-world problems and indicated its use in other application domains.

\section{ACKNOWLEDGEMENTS}

The authors place on record their thanks to the authorities of Gudlavalleru Engineering College, A.P for the facilities they provided.

\section{REFERENCES}

[1] A Granular Reflex Fuzzy Min-Max Neural Network for Classification (GrRFMN) by A.V.Nandedkar, P. K. Biswas, IEEE Trans. on Neural Networks, 20, pp. 11171134 (2009).

[2] A Fuzzy Min-Max Neural Network Classifier with Compensatory Neuron (FMCN) Architecture by Nandedkar A. V. and Biswas P. K., IEEE Trans. on Neural Network, Vol 18(1), pp. $42-54$ (2007).

[3] B.Gabrys and A. Bargiela, "General fuzzy min-max neural network for clustering and classification," IEEE 
Trans. Neural Netw., vol.11, no. 3, pp. 769-783, May 2000 .

[4] W. Pedrycz," and G. Vukovich, "Granular Computing in Pattern Recognition," in Neuro-Fuzzy Pattern Recognition, H. Bunke and A. Kandel, Eds. Singapore: World Scientific, 2000, pp. 25-143.

[5] L. A. Zadeh, "Towards a theory of fuzzy information granulation and its centrality in human reasoning and fuzzy logic Fuzzy Sets Syst., vol.90, pp. 111-127, 1997.

[6] A. V. Nandedkar and P. K. Biswas, "A reflex fuzzy min max neural network for granular data classification," in Proc. 18th Int. Conf. Pattern Recognit., Hong Kong, 2006, vol. 2, pp. 650-653.
[7] W.Pedrycz, "Genetic tolerance fuzzy neural networks: From data to fuzzy hyperboxes," Neurocomputing, vol. 70, no. 7-9, pp. 1403-1413, Mar. 2007.

[8] A. Bargiela and W. Pedrycz, "Classification and clustering of granular data," in Proc. Joint IFSA World Congr./20th Int. Conf. NAFIPS, Jul. 2001, vol. 3, pp. 1696-1701.

[9] W. Pedrycz and G. Vukovich, "Granular neural networks,” Neurocomputing,vol. 36, pp. 205-224, 2001.

[10] P. K. Simpson, "Fuzzy min-max neural network-Part I: Classification,"IEEE Trans. Neural Netw., vol. 3, no. 5, pp. 776-786, Sep. 1992. 